\title{
Discovery of Clinically Relevant Fusions in Pediatric Cancer
}

Peter White ${ }^{1,3, *}$

*Corresponding Author: Prof. Peter White, PhD, The Steve and Cindy Rasmussen Institute for

Genomic Medicine, Nationwide Children's Hospital, 575 Children's Crossroad, Columbus, OH 43215. USA. Tel: +1 (614) 355-2671; Email: peter.white@nationwidechildrens.org

Keywords: transcriptomics, genomics, pediatric neoplasms, gene fusions, cancer, RNA-Seq

Running title: Fusion Identification in Pediatric Cancer 
ABSTRACT

Background: Pediatric cancers typically have a distinct genomic landscape when compared to adult cancers and frequently carry somatic gene fusion events that alter gene expression and drive tumorigenesis. Sensitive and specific detection of gene fusions through the analysis of nextgeneration-based RNA sequencing (RNA-Seq) data is computationally challenging and may be confounded by low tumor cellularity or underlying genomic complexity. Furthermore, numerous computational tools are available to identify fusions from supporting RNA-Seq reads, yet each algorithm demonstrates unique variability in sensitivity and precision, and no clearly superior approach currently exists. To overcome these challenges, we have developed an ensemble fusion calling approach to increase the accuracy of identifying fusions.

Results: Our ensemble fusion detection approach utilizes seven fusion calling algorithms: Arriba, CICER0, FusionMap, FusionCatcher, JAFFA, MapSplice, and STAR-Fusion, which are packaged as a fully automated pipeline using Docker and AWS serverless technology. This method uses paired end RNA-Seq sequence reads as input, and the output from each algorithm is examined to identify fusions detected by a consensus of at least three algorithms. These consensus fusion results are filtered by comparison to an internal database to remove likely artifactual fusions occurring at high frequencies in our internal cohort, while a "known fusion list" prevents failure to report known pathogenic events. We have employed the ensemble fusion-calling pipeline on RNA-Seq data from 229 patients with pediatric cancer or blood disorders studied under an IRB-approved protocol. The samples consist of 138 central nervous system tumors, 73 solid tumors, and 18 hematologic malignancies or disorders. The combination of an ensemble fusion-calling pipeline and a knowledge-based filtering strategy identified 67 clinically relevant fusions among our 47 cohort (diagnostic yield of 29.3\%), including RBPMS-MET, BCAN-NTRK1, and TRIM22-BRAF 
48 fusions. Following clinical confirmation and reporting in the patient's medical record, both known

49 and novel fusions provided medically meaningful information.

50 Conclusions: Our ensemble fusion detection pipeline offers a streamlined approach to discover

51 fusions in cancer, at higher levels of sensitivity and accuracy than single algorithm methods.

52 Furthermore, this method accurately identifies driver fusions in pediatric cancer, providing

53 clinical impact by contributing evidence to diagnosis and, when appropriate, indicating targeted

54 therapies.

\section{BACKGROUND}

Globally, there are approximately 300,000 pediatric and adolescent cases of cancer

diagnosed each year [1, 2]. While advances in medicine have led to a drastic improvement in 5-

year overall survival rates (up to $84 \%$ in children under 15), pediatric cancer remains the most

common cause of death by disease in developed countries $[3,4]$. Pediatric cancers are defined by a

61 distinct genomic landscape when compared to adult cancers, which includes an overall low

62 number of somatic single nucleotide variants, common driver fusions and epigenetic changes that

63 drive a specific transcriptional program. Pediatric cancers are often considered embryonic in

64 origin and demonstrate a significant germline predisposition component approaching 10\% [5-7].

Many pediatric tumors contain gene fusions resulting from the juxtaposition of two genes

66 (AdDitional FILE 1: FiguRE S1)[6]. Fusions typically occur through chromosomal rearrangements,

67 and often lead to dysregulated gene expression of one or both gene partners [8-11]. Fusions can

68 also generate chimeric oncoproteins, wherein functional domains from both genes are retained,

69 often leading to aberrant and strong activation of nonspecific downstream targets [12]. The

70 alterations in gene expression and activation of downstream targets induced by fusions are 
71 considered to be oncogenic events in pediatric cancer and increasingly may indicate response to

72 specific targeted therapies.

The identification of an oncogenic fusion can provide medically meaningful information in

the context of diagnosis, prognosis, and treatment regimens in pediatric cancers. Fusions may provide diagnostic evidence for a specific histological subgroup. For example, EWSR1-FLI1 fusions subgrouping supratentorial ependymomas [12]. The detection of certain fusions, such as $B C R-A B L$

in acute lymphocytic leukemia, can be used as a surrogate for residual tumor load and treatment

response [13]. Fusions may also provide prognostic indication, such as KIAA1549-BRAF in low

grade astrocytomas, which have a more favorable outcome compared to non- $B R A F$ fused tumors

$[14,15]$. In addition, fusions that involve kinases can present therapeutic targets, including FGFR1-

TACC1, FGFR3-TACC3, NPM1-ALK, and NTRK fusions [2, 12, 16-19].

However, regardless of the clear clinical benefits of characterizing fusion events in a given

alone is not straightforward and they often go undiscovered. In particular, many fusions are not coding or intronic regions which may not have corresponding capture probes. Even whole genome sequencing (WGS) NGS data has proved difficult to evaluate complex rearrangements resulting in gene fusions due to a high false positive rate and due to the limitations of short read lengths [20,

91 unbiased data type suitable for fusion detection, while also providing information about the

92 expression of fusion transcripts, including multiple isoforms, and fusions that occur due to 93 aberrant splicing events [22, 23]. 
While RNA-Seq is a powerful tool for fusion detection, it is not without its limitations.

Notably, there is currently a major deficit in our ability to accurately identify fusions in spite of

having many computational approaches available. Here, consistently identifying gene fusion

events with high sensitivity and precision using one algorithm is unlikely and this is of critical

importance in a clinical diagnostic setting [12]. Computational approaches that have been tuned

for high sensitivity are limited by also calling numerous false positives, requiring extensive

manual review of data, while those with a low false discovery rate (FDR) often miss true positives

101 due to over-filtering [12]. To overcome these complications of sensitivity and specificity, we have

102 employed an ensemble pipeline, which merges results from seven algorithmic approaches to

103 identify, filter and output prioritized fusion predictions.

Another common issue encountered in fusion prediction is the identification of likely non-

pathogenic fusions, due both to read-through events and fusions occurring in non-disease

involved (normal) genomes.[12, 24, 25] We addressed these sources of false positivity through the

implementation of a filtering strategy that removes known normal fusions and RNA transcription

read-through events, based on internal frequency of detection and location of chromosomal

breakpoints. Lastly, to prevent over-filtering and inadvertent removal of previously described known pathogenic fusion events, we have developed and continually update a list containing

111 known pathogenic fusion partners, that will return any data-supported fusions to the output list of 112 prioritized fusion results for further evaluation.

The ensemble fusion detection pipeline outperformed all single algorithm methods we 114 evaluated, achieving high levels of sensitivity, while simultaneously minimizing false positive calls 115 and non-clinically relevant fusion predictions. Here, we describe our ensemble fusion detection 116 approach, its performance on commercial control reference standards with known fusions, and its 117 implementation on a pediatric cohort consisting of rare, treatment refractory, or relapsed cancers 
118 and hematologic diseases. Utilization of our ensemble approach resulted in a diagnostic yield of

119 approximately $30 \%$ in our cohort, identified novel fusion partners, and has provided diagnostic

120 information and/or targeted treatment options for this patient population.

\section{RESULTS}

Development and optimization of ensemble pipeline on a control reference standard

Identification of gene fusions through the use of a single algorithm is often associated with

approaches to improve upon these deficits, we studied the intricacies of several fusion detection

true positive fusions while minimizing false positive fusions [25-27]. After evaluating each consensus calls from seven different computational approaches (FIGURE 1A), calculates the

131 concordant fusion partners and breakpoints, and filters this output list based on internal

132 frequency, reads of evidence, and breakpoint location. A list of known pathogenic fusions rescues

133 any known pathogenic fusion gene partners with suitable algorithmic and read support for further 134 evaluation (AdDitionAL FILE 1: TABLE S3).

To optimize the approach, we utilized a reference standard from a commercial provider 136 (Seraseq Fusion RNA, SeraCare, Milford, MA), containing synthetic RNAs representing 14 cancer137 associated fusions in varying proportions (ADDITIONAL FILE 1: TABLES S1 AND S2). Data generated 138 from these RNA-Seq libraries, performed as replicates for a range of dilutions, were analyzed 139 using the ensemble pipeline. We compared the output derived from a consensus of two or more 140 callers to that from a consensus of three or more callers by calculating sensitivity (\# of Seraseq 141 fusions identified)/(14 possible Seraseq fusions), and precision (\# of Seraseq fusions 
142 identified)/(\# of total fusions identified) prior to filtering or known fusion list comparison. The

143 undiluted reference standard with consensus of at least two callers, had a sensitivity of $100 \%$ and

144 precision of $36.36 \%$. Inclusion of the knowledgebase filtering step reduced the sensitivity to

$14585.71 \%$ while increasing the precision to $77.42 \%$, and the known fusion list rescue step increased

146 sensitivity to $100 \%$ and precision to 80\% (ADDITIONAL FILE 1: TABLE S5, FiguRE S6A). By increasing

147 the consensus requirement to three callers, rather than just two, the prefiltered sensitivity was

$148100 \%$ and precision was $93.33 \%$. Inclusion of the filtering step reduced the sensitivity to $85.71 \%$

149 while increasing the precision to $100 \%$, and known fusion list rescue increased sensitivity to

150 100\% and precision to 100\% (TABLE 2; ADDITIONAL FILE 1: FIGURE S6A). The inclusion of the known

151 fusion list prevented the removal of known Seraseq fusions, due to too few reads of evidence or 152 number of callers providing support, as well as a single Seraseq fusion, EML4-ALK, which was 153 present at an artificially high frequency in our database (24.7\%) due to false positive calls by 154 FusionCatcher. Implementation of the known fusion list led to sensitivity scores of $100 \%$ for both 155 levels of caller consensus. The individual fusion detection algorithms ranged in sensitivity and 156 precision, and while certain algorithms are able to maintain high levels of sensitivity in addition to 157 moderate levels of precision, such as STAR-Fusion (sensitivity $=100 \%$, precision $=43.75 \%$ ), 158 others such as FusionCatcher (sensitivity $=92.86 \%$, precision $=4.34 \%)$ and CICERO $($ sensitivity $=$ 159 100\%, precision $1.06 \%$ ) had high levels of sensitivity with very low precision levels (TABLE 2; 160 Additional File 2: TABLE S5). When considering the overall results from undiluted and serial 161 dilutions of the reference standard, the required overlap of at least three callers, with filtering and 162 utilization of the known fusion list, led to significantly fewer total fusions identified compared to 163 two consensus callers ( $\mathrm{p}=1.86 \mathrm{E}-07$ )(Table 2; Additional File 1: Figure S6B, Table S6). The 164 ensemble pipeline results obtained from various reference standard dilutions, with a minimum of 165 three callers in consensus, using filtering and known fusion list rescue are shown (FIGURE 1B; 
AdDitional FiLE 2: TABLE S5). The optimized ensemble pipeline, consisting of a consensus of three

167 callers, filtering, and the known fusion list, maintained high levels of sensitivity, (at least 90.48\%),

168 while maintaining $100 \%$ precision as low as the $1: 50$ dilution of the reference standard

169 (AdDitional File 2: TABLE S5). In addition to the high levels of sensitivity and precision, the total

170 number of fusions identified by this optimized ensemble pipeline in undiluted and diluted samples

171 was significantly fewer than the number identified by individual fusion detection algorithms,

172 including STAR-Fusion $(\mathrm{p}=1.77 \mathrm{E}-12)$, CICERO $(\mathrm{p}=3.39 \mathrm{E}-14)$ and FusionCatcher $(\mathrm{p}=1.00 \mathrm{E}-$

173 08)(Additional File 1, Table S6). These results highlights the removal of false positive fusions,

174 which includes artifactual and benign fusion events, and subsequent reduction in manual

175 evaluation requirements (ADDiTionAL FILE 1: FiguRE S6C,D). Notably, we only considered the 14

176 Seraseq synthetic fusions as true positives. While fusions may exist within the GM24385 cell line,

177 in the optimized ensemble approach all of these fusions were filtered out due to either high

178 frequency across our cohort or supporting read evidence below our minimum threshold, 179 suggesting that they are likely to be artifactual in nature.

181 Implementation of the ensemble approach on an in-house pediatric cancer and hematologic disease 182 cohort

Having demonstrated the efficacy of the optimized ensemble fusion detection pipeline using synthetic fusion samples, we further evaluated the utility of the pipeline on RNA-Seq data obtained from 229 patient samples, obtained from three prospective pediatric cancer and 186 hematologic disease studies at Nationwide Children's Hospital (NCH) (ADDITIONAL FILE 1: FIGURE 187 S2). Our ensemble pipeline identified significantly fewer total predicted fusions post-filtering, 188 compared to all other single callers (FIGURE 2A; AdDITIONAL FILE 1: TABLE S7). Applying the known 189 fusion list rescue altered the average number of fusions identified overall, as an average of 3.88 
190 fusions per case were identified by 3 or more callers, while an average of 3.93 fusions were

191 identified by 3 or more callers after applying the known fusion list; a total of 11 fusions were

192 rescued by this approach, of which 1 (KIAA1549-BRAF; ADDITIONAL FILE 3: TABLE S8) was clinically

193 relevant . The retained KIAA1549-BRAF fusion was identified by three callers, but was initially

194 filtered out due to too few reads of evidence, possibly due to either low expression, low tumor

195 cellularity or clonality (FIGURE 2D). In total, 67 clinically relevant fusions, identified in 67 different

196 cases, (33 CNS, 7 heme, and 27 solid tumor; ADDITIONAL FILE 1: FIGURE S7) were discovered using

197 the optimized ensemble pipeline with automated filtering, including the known fusion list feature,

198 and a consensus of three callers (29.3\% of tumors contained a clinically relevant fusion).

199 Regardless of source material, there was a roughly a 30\% yield; with clinically relevant fusion

200 identification in 44 of 148 frozen samples (30\% yield), 19 of 68 FFPE samples (28\% yield), and 4

201 of 13 other samples (31\% yield), which included blood, cerebral spinal fluid, or bone marrow

202 (AdDitional File 1: Figure S7). No single fusion detection algorithm was able to identify all 67

203 fusions. While JAFFA was the most sensitive algorithm, identifying the most clinically relevant

204 fusions (64 out of 67), it also had one of the highest average numbers of fusions identified per

205 sample, 1409 fusions, indicating a large number of likely false positives (FIGURE 2B; ADDITIONAL

206 FILE 1: TABLE S7). Identified fusions were broken down into 4 types: Interchromosomal Chimeric

$207(n=30)$, Intrachromosomal Chimeric $(n=29)$, Loss of Function $(n=3)$, and Promoter Swapping $(n=$

208 5)(FIGURE 2C). Of the 67 clinically relevant fusions, seven were considered novel events, defined as

209 a gene fusion involving two partners not previously described in the literature at the time of

210 identification (FIGURE 2D). Of the 67 fusions detected, 40 (60\%) were identified by all seven

211 callers, $55(82 \%)$ were identified by $\geq 6$ callers, 60 (90\%) were identified by $\geq 5$ callers, 64 (96\%)

212 were identified by $\geq 4$ callers, and 67 (100\%) were identified by $\geq 3$ callers. (FIGURE 2E). One

213 sample experienced an unresolvable failure of FusionMap, likely due to high sequencing read 
214 number. Results from the remaining callers, which successfully completed for this sample, were

215 still included in our analysis. These results highlight the ability of the optimized ensemble

216 approach to identify gene fusions with a high level of confidence and a reduced number of false

217 positive predictions, while preventing over-filtering by comparison to a list of known pathogenic

218 fusions.

Clinical Impact of Fusion Prediction

221 An RBPMS-MET fusion in an infantile fibrosarcoma-like tumor

tumor consisted of variably cellular fascicles of spindle cells with a nonspecific

224 immunohistochemical staining profile, suspicious for infantile fibrosarcoma. However, the tumor

was negative for an ETV6-NTRK3 fusion, one of the defining features of infantile fibrosarcoma [28].

RNA-Seq of the primary tumor and optimized ensemble pipeline analysis revealed an $R B P M S-M E T$

227 fusion as the only consensus call. By contrast, the individual callers identified numerous fusions as

228 follows: Arriba: 16, CICER0: 2142, FusionMap: 29, FusionCatcher: 3907, JAFFA: 1130, MapSplice:

229 18, and STAR-Fusion: 20 (Figure 3A, Additional File 3: Table S8). RBPMS, an RNA-binding

230 protein, and $M E T$, a proto-oncogene receptor tyrosine kinase, have been identified as fusion

231 partners in a variety of cancers with other genes and as gene fusion partners in a patient with

232 cholangiocarcinoma [29]. Although MET fusions are uncommon drivers of sarcoma [30], a TFG-

233 MET fusion has been reported in a patient with an infantile spindle cell sarcoma with neural 234 features [31-33]. The interchromosomal in-frame fusion of RBPMS (NM_006867, exon 5) to MET 235 (NM_000245, exon 15) juxtaposes the RNA recognition motif of RBPMS to the MET tyrosine kinase 236 catalytic domain (FIGURE 3B,C). Given the therapeutic implications of this driver fusion, the fusion 237 was confirmed and reported in the patient's medical record. The identification of this fusion 
238 provided the molecular driver for this tumor, which enabled definitive classification as an infantile

239 fibrosarcoma-like tumor with a MET fusion. The patient was initially treated with VAC (vincristine,

240 actinomycin D, and cyclophosphamide) chemotherapy which reduced tumor burden. Surgical

241 resection of the mass was performed with positive margins. Given the presence of a targetable

242 gene fusion, the presence of residual tumor, and the morbidity associated with additional surgery

243 or radiation, the patient was subsequently treated with the MET inhibitor cabozantinib and

244 demonstrated a complete pathological response (FIGURE 3D).

An NTRK1 fusion in an infiltrating glioma/astrocytoma

A 6-month-old female was diagnosed with an infiltrating glioma/astrocytoma, with a 248 mitotic index of 7 per single high-power field (HPF) and a Ki-67 labeling index averaging nearly $24920 \%$, indicative of aggressive disease. RNA-Seq of the primary tumor revealed a BCAN-NTRK1 250 fusion, identified by five callers as the only consensus fusion output from the optimized ensemble 251 pipeline (FIGURE 4A). This fusion was clinically confirmed by RT-PCR as an in-frame event, 252 resulting from an intrachromosomal deletion of $225 \mathrm{~kb}$ at $1 \mathrm{q} 23.1$, which juxtaposes $B C A N$ 253 (NM_021948, exon 6) to NTRK1 (NM_002529, exon 8) (FIGURE 4B,C). This fusion results in the loss 254 of the ligand binding domain of NTRK1, while retaining the tyrosine kinase catalytic domain, 255 leading to a predicted activation of downstream targets in a ligand-independent manner [34]. 256 Comparison of the normalized read counts from RNA-Seq data revealed elevated NTRK1 257 expression, over 7 standard deviations from the mean, relative to NTRK1 expression for CNS 258 tumors within the NCH cohort (N=138) (FIGURE 4D). This result indicates the use of first 259 generation TRK inhibitor therapies, with recent regulatory approvals, that have exemplary 260 response rates (75\%) and are generally well tolerated by patients [34]. Although the patient has 261 no evidence of disease following gross total resection and treatment with conventional 
262 chemotherapy, TRK inhibitors may be clinically indicated in the setting of progressive disease 263 given these findings (FIGURE 4E).

Novel BRAF fusion in a mixed neuronal-glial tumor

neuronal-glial tumor of unusual morphologic appearance. Tumor histology had features of both

ganglioglioma and pilocytic astrocytoma. This tumor was negative for the somatic variant $B R A F$

p.V600E, one of the most common somatic alterations associated with gangliogliomas and

pilocytic astrocytomas [35]. Both the ganglioglioma and pilocytic astrocytoma-like portions of the

271 primary tumor were studied separately by RNA-Seq. A novel TRIM22-BRAF fusion was identified

272 in both histologies of the tumor, with fusion overlap results from the ganglioglioma portion

273 represented in FIGURE 5A. TRIM22-BRAF was the only consensus fusion output by the optimized

274 fusion detection pipeline, and was clinically confirmed by RT-PCR. TRIM22 and BRAF are novel

275 fusion partners; however, TRIM22 has been reported with other fusion partners in head/neck

276 squamous cell carcinoma [36]. BRAF is a known oncogene that activates the RAS-MAPK signaling

277 pathways, and has been described with numerous fusion partners, including the common

278 KIAA1549-BRAF fusion in pediatric low-grade gliomas [35]. This fusion is an interchromosomal

279 translocation occurring between TRIM22 (NM_006074, exon 2) at 11p15.4 and BRAF

280 (NM_004333, exon 9) at 7q34. The resulting protein includes the TRIM22 zinc finger domains and

281 the BRAF tyrosine kinase domain (FIGURE 5B,C). The TRIM22-BRAF fusion may lead to constitutive

282 dimerization and activation of BRAF kinase domain, which is indicated by single sample Gene Set

283 Enrichment Analysis (ssGSEA) and is theoretically targetable through RAF, MEK, or mTOR 284 inhibitors (FIGURE 5D,E). 


\section{DISCUSSION}

Fusions play a significant role as common oncogenic drivers of pediatric cancers, and their

288 identification may refine diagnosis, inform prognosis, or indicate potential response to molecularly targeted therapies. We have developed an optimized pipeline for fusion detection that harmonizes results from several fusion calling algorithms, filters the output to remove known

291 false positive results, and evaluates the detected fusions compared to a list of known pathogenic 292 fusions. Testing this pipeline on a reference standard indicated that it outperforms single fusion 293 detection algorithms by reducing the number of false positive calls, producing a smaller number of 294 fusions prioritized by the strength of supporting evidence, and suitable for manual inspection. As such, our pipeline greatly simplifies the interpretation process, enabling our multidisciplinary oncology teams to focus on medically relevant findings. and hematologic disease cases and identified 67 fusions. Of these, the fusions from 50 patients were selected for clinical confirmation by an orthogonal method, in our CAP-accredited, CLIAvalidated clinical laboratory. All 50 (100\% true positive rate) were confirmed to be true fusion

301 events, and were determined to be of clinical relevance by our multidisciplinary care team, 302 providing a diagnostic yield of over 29\% across the cohort. (ADDITIONAL FiLE 3: TABLE S8). Given 303 the high number of putative fusions observed with any single caller, it can be difficult to manually 304 identify a pathogenic fusion amongst a list of tens, if not hundreds, of output fusions. By taking 305 into consideration the frequency in which each fusion occurs in an internal database, as well as the 306 level of evidence based on the number of callers and number of supporting reads by each caller, 307 one can more confidently remove false positives and identify relevant fusions. While our approach 308 does not remove the necessity of manual curation, which is required to determine true clinical 309 relevance of a fusion, it is able to drastically reduce the number of fusions that must be manually 
310 assessed, down to $\sim 4$ fusions per case, and provides annotations, including a pathogenicity gene

311 partner score, to ease manual interpretation efforts. Our fully automated pipeline aids in

312 prioritization, filtering, and subsequent knowledge-based analysis, providing a more streamlined

313 and less labor-intensive approach to identify fusions, compared to current fusion identification

314 methodologies, drastically reducing the manual workload required to sort through unfiltered or

315 unprioritized results.

316 The most frequent fusion identified within our pediatric cancer cohort was KIAA1549-BRAF

$317(\mathrm{n}=12$, frequency $=5.2 \%$; FIGURE 2B)[17]. This fusion is characteristically found in pilocytic

318 astrocytomas, which comprise $8.7 \%$ of our pediatric cancer cohort (20 out of 229 cases)[37]. We

319 identified five different sets of KIAA1549-BRAF breakpoints within our cohort (ADDITIONAL FILE 1:

320 FIGURE S8A). The most common fusion patterns represented in the literature are KIAA1549 exon

321 16-BRAF exon 9 (16-9) or KIAA1549 exon 15-BRAF exon 9 (15-9), and these two breakpoints

322 represent 9 of the 12 KIAA1519-BRAF fusions we identified (ADDITIONAL FILE 1: FIGURE S8B) [38,

323 39]. Three additional previously described sets of breakpoints were also identified, KIAA1549

324 exon 16-BRAF exon $11(16-11 ; \mathrm{n}=1)$, KIAA1549 exon 15-BRAF exon 11 (15-11; n=1), and KIAA1549

325 exon 13-BRAF exon 9 (13-9; n=1; Additional File 1: Figure S8). While the 16-11 and 15-11

326 breakpoints occur less frequently than $16-9$ or $15-9$, they have been well described in the

327 literature [38]; whereas only a single case with 13-9 breakpoints was reported as part of a 328 pilocytic astrocytoma cohort study [40]. KIAA1549-BRAF fusions often have low levels of 329 expression, a phenomenon that has been described in the literature and is associated with 330 difficulties in its identification through RNA-Seq based methodologies, which lack fusion product 331 amplification [41]. The ability of the ensemble pipeline to identify KIAA1549-BRAF fusions, and 332 others that have very low levels of expression, highlights the sensitivity of our approach. 333 Additionally, a supplementary "singleton" file for fusions that are identified by individual 
334 algorithms and on the known fusion list is also output by our approach, allowing users the 335 opportunity to manually interpret singleton results. This approach ensures that fusions on the 336 known fusion list are retained, even with minimal evidence by a single caller.

Our approach has also identified other fusions commonly associated with pediatric cancer,

338 including EWSR1-FLI1 (n=9), FGFR1-TACC1 (n=3), PAX3-FOXO1 (n=3), C11orf95-RELA (n=2),

339 COL3A1-PLAG1 (n=2), and NPM1-ALK (n=2) (FIGURE 2B). In addition to common fusions, our 340 ensemble pipeline also identified seven novel fusions (FIGURE 2B). Five of the seven novel fusions

341 were confirmed by an orthogonal assay in our clinical lab (AdDITIONAL FiLE 3: TABLE S8). Chimeric

342 fusions, which include both interchromosomal $(n=30)$ and intrachromosomal $(n=29)$ events, were

343 the most common type of fusion identified within the cohort, however, 5 promoter swapping and

3443 loss of function fusions were also identified, highlighting the range of fusions this approach is 345 able to detect (FIGURE 2D).

346 Running seven different fusion callers is computationally complex, as each has its own set

347 of dependencies and environmental requirements. To overcome this, we utilize modern cloud 348 computing technologies. Most notable, our entire pipeline has been built in an AWS serverless 349 environment, removing the requirement for high performance computing (HPC) clusters, while 350 producing highly reproducible results and enabling pipeline sharing. The use of a serverless 351 environment provides flexibility to deploy and scale applications regardless of the application's 352 size, without needed concern for the underlying infrastructure. We are also leveraging containers 353 to process the data within the serverless environment, as they can be easily utilized by outside 354 institutions with little to no adjustment to their own environments. Another benefit to the current 355 structure of our approach is the ability to assess output from the individual algorithms in real 356 time, as the ensemble pipeline is automatically run after each individual caller completes, allowing 357 for interpretation of at least 3 of the 7 callers within $\sim 3.5$ hours, which can be beneficial in 
358 situations that necessitate fast turnaround times (ADDITIONAL FILE 1: FiguRE S5). Overall, our novel

359 use of serverless technology provides a robust computational solution that is fully automatable 360 and easy to distribute.

There are numerous benefits to the utilization of this optimized pipeline, in that detected

362 fusion events are agnostic to gene partner, allowing identification of common, rare and novel

363 fusions. In addition, the RNA-Seq data set can be utilized for other types of downstream and 364 correlative analyses, including evaluation of gene expression for loci disrupted by the fusion 365 (FIGURE 4D). Utilization of cohort data to assess outlier gene expression can provide valuable 366 insights into pathway disruptions that may occur due to the gene fusion (FIGURE 5D) and may 367 provide information about disease subtyping. and which callers to deploy. This may impact potential cost savings, time-to-result, or permit customization that eliminates specific callers that require excessive compute requirements or run times, as suitable in a clinical diagnostic or research setting. Users can also determine the number of consensus calls required to support fusion prediction, which can reduce the number of fusions to assess manually. Callers with a higher percentage of false positives, FusionCatcher and JAFFA, often overlap in their predictions, leading to an increased average number of fusions output by the ensemble pipeline with a consensus of only two callers; a problem diminished by requiring predictions from at least three callers to overlap. In our study, precision was found to be highest in the three-caller consensus version of the ensemble pipeline (TABLE 2; Additional File 2: TABLE S5). Another benefit to utilizing different algorithms is the ability to assess supplementary output data, in addition to traditional fusion calling. We have made use of this through the inclusion of the internal tandem duplication (ITD) detection which is performed by CICERO. CICERO has identified 
3817 clinically relevant ITDs within our cohort, 4 of which we have confirmed using orthogonal assays

(ADDiTIONAL FILE 1: TABLE S9).

Future developments to the pipeline could include a weighting system for each caller, based on the precision and sensitivity of the algorithm and on which callers have overlapping predictions, leading to a more sophisticated prioritization strategy. Additional fusion calling algorithms may also be considered and provided as options for users. The known fusion list can also be modified and tailored to include specific gene pairs, or even single genes of interest, providing another layer of customization. Importantly, through the utilization of a proper internal database for frequency filtering purposes, considering age and/or cancer diagnosis, and with the deployment of the appropriate known fusion list, the ensemble approach could be readily 391 implemented in adult cancer fusion detection. Lastly, not all predictors performed equally, and 392 there was a single unresolvable failure of FusionMap to complete. This failure was likely due to the 393 sequencing depth of the sample, however further analysis is required to determine whether 394 parameter modification would permit completion of FusionMap in this case (ADDITIONAL FILE 3: 395 TABLE S8). Importantly, our approach was able to circumvent this failure due to the multi-caller 396 nature of the pipeline. Lastly, there are many modalities of RNA-seq analysis that may be 397 harnessed in future developments of the ensemble fusion detection pipeline, which may include 398 an integrative approach exploiting expression-based analysis and ranking. In summary, the 399 ensemble pipeline provides a highly customizable approach to fusion detection that can be applied 400 to numerous settings, with opportunities for future improvements based on additional features 401 and applications.

\section{Conclusions:}


The optimized ensemble fusion detection pipeline provides a highly automated and accurate approach to fusion detection, developed to identify high confidence gene fusions from 406 RNA-Seq data produced from pediatric cancer and hematologic disease samples, and could be 407 readily implemented in adult cancer data analysis. The clinical impact of accurately identifying 408 gene fusions in a given patient's tumor sample is undeniable, not only in terms of refining 409 diagnoses but also in terms of providing prognostic information that shapes treatment decisions. 410 Furthermore, identification of driver fusions may indicate potential response to targeted therapies 411 for cancer patients. The code for the overlap algorithm utilized in this study is publicly available at 412 our GitHub page (https://github.com/nch-igm/nch-igm-ensemble-fusion-detection).

\section{METHODS}

415 Description of an internal patient cohort

416 In total, 229 patients were consented and enrolled onto one of three Institutional Review 417 Board (IRB) approved protocols (IRB17-00206, IRB16-00777, IRB18-00786) and studied at the 418 Institute for Genomic Medicine (IGM) at Nationwide Children's Hospital (NCH) in Columbus, Ohio. 419 Through the utilization of genomic and transcriptomic profiling, these protocols aim to refine 420 diagnosis and prognosis, detect germline cancer predisposition, identify targeted therapeutic 421 options, and/or to determine eligibility for clinical trials in patients with rare, treatment422 refractory, relapsed, pediatric cancers or hematologic diseases, or with epilepsy arising in the 423 setting of a low grade central nervous system (CNS) cancer. Our in-house NCH cohort as studied 424 here, consisted of samples from CNS tumors $(n=138)$, hematologic diseases ( $n=18)$, and non-CNS 425 solid tumors ( $\mathrm{n}=73$ ), as represented in AdDITIONAL FILE 1: FigURE S2. 
RNA was extracted from snap frozen tissue, formalin-fixed paraffin-embedded (FFPE) tissue, peripheral blood, bone marrow, and cerebral spinal fluid utilizing dual RNA and DNA coextraction methods originally developed by our group for The Cancer Genome Atlas project [42].

431 White blood cells were isolated from peripheral blood or bone marrow using the lymphocyte 432 separation medium Ficoll-histopauqe. Frozen tissue, white blood cells, or pelleted cells from 433 cerebrospinal fluid were homogenized in Buffer RLT, with beta-Mercaptoethanol to denature 434 RNases, plus Reagent DX and separated on an AllPrep (Qiagen) DNA column to remove DNA for 435 subsequent RNA steps. The eluate was processed for RNA extraction using acid-phenol:chloroform 436 (Sigma) and added to the mirVana miRNA (Applied Biosystems) column, washed, and RNA was 437 eluted using DEPC-treated water (Ambion). DNAse treatment (Zymo) was performed post RNA 438 purification. FFPE tissues were deparaffinized using heptane/methanol (VWR) and lysed with 439 Paraffin Tissue Lysis Buffer and Proteinase K from the HighPure miRNA kit (Roche). The sample 440 was pelleted to remove the DNA, and the supernatant was processed for RNA extraction with the 441 HighPure miRNA column, followed by DNase treatment (Qiagen). RNA quantification was 442 performed with Qubit (Life Sciences).

RNA-Seq libraries were generated using $100 \mathrm{ng}$ to $1 \mu \mathrm{g}$ of DNase-treated RNA input, either 444 by ribodepletion using the Ribo-Zero Globin kit (Illumina) followed by library construction using 445 the TruSeq Stranded RNA-Seq protocol (Illumina), or by ribodepletion with NEBNext 446 Human/Mouse/Rat rRNA Depletion kit followed by library construction using the NEBNext Ultra 447 II Directional RNA-Seq protocol (New England BioLabs). Illumina 2x151 paired end reads were 448 generated either on the HiSeq 4000 or NovaSeq 6000 sequencing platforms (Illumina). An average 449 of 104 million read pairs were obtained per sample (range 37M to 380M read pairs).

450 Following data production and post-run processing, FASTQ files were aligned to the 451 GRCh38 human reference (hg38) using STAR aligner (version 2.6.0c)[43]. Feature counts were 
452 calculated using HTSeq, and normalized read counts were calculated for all samples using DESeq2 $453[44,45]$. Single sample Gene Set Enrichment Analysis (ssGSEA), v10.0.3, was performed on 454 DESeq2 normalized read counts using Molecular Signatures Database (MSigDB) Oncogenic 455 Signatures (c6.all.v7.2.symbols.gmt), which included MEK-upregulated genes (MEK_UP.V1_UP), 456 RAF-upregulated genes (RAF_UP.V1_UP), and mTOR-upregulated genes 457 (MTOR_UP.N4.V1_UP)[46].

RNA-Seq of SeraCare control reference standards

Seraseq Fusion RNA Mix (SeraCare Inc., Milford, MA) was utilized as a control reference 461 standard reagent to test and optimize the ensemble fusion detection pipeline. This product 462 contains 14 synthetic gene fusions in vitro transcribed, utilizing the GM24385 cell line RNA as a 463 background. RNA-Seq libraries were prepared utilizing 500 ng input of neat (undiluted) Seraseq 464 Fusion RNA v2, a non-commercially available concentrated product, as input (SeraCare). RNA-Seq 465 libraries were also prepared using 500 ng input of diluted control reference standard (Seraseq 466 Fusion RNA v3 (SeraCare)), which, as a neat reagent is roughly equivalent to a 1:25 dilution of the 467 v2 product, and of total human RNA (GM24385, Coriell) for the fusion-negative controls. 468 Concentrations of individual fusions in the control reference standard were determined by the 469 manufacturer using a custom fluorescent probe set (based on TaqMan probe design) for each 470 fusion and evaluation by droplet digital PCR. Digital PCR-based concentration data (copies/ul) are 471 available in Additional File 1: TABLE S1 for the undiluted sample and Additional FiLE 1: TABLE S2 472 for the diluted sample [47]. Dilutions of the Seraseq Fusion RNA v3 reference standard were performed by mixing with 474 control total human RNA (GM24385, Coriell) for final dilutions of 1:25, 1:50, 1:250, 1:500, 1:2500. 475 We also evaluated undiluted Seraseq Fusion RNA v2. For neat and diluted samples, 500ng input 
476 RNA was treated using the NEBNext Human/Mouse/Rat rRNA Depletion kit and libraries were 477 prepared following the NEBNext Ultra II Directional RNA-Seq protocol (New England BioLabs). 478 Paired end $2 \times 151 \mathrm{bp}$ reads were produced using the HiSeq 4000 platform (Illumina). An average 479 of 149 million read pairs were obtained per Seraseq sample (range of 86M to 227M read pairs).

Fusions were detected from paired end RNA-Seq FASTQ files utilizing an automated

TABLE 1: Arriba (v1.2.0), CICER0 (v0.3.0), FusionMap (v mono-2.10.9), FusionCatcher (v0.99.7c), JAFFA (direct v1.09), MapSplice (v2.2.1), and STAR-Fusion (v1.6.0)[25, 48-51]. STAR-Fusion parameters were altered to reduce the stringency setting for the fusion fragments per million reads (FFPM) from 0.05 to 0.02 , while default parameters were retained for all other callers. After fusion calling with each independent algorithm, a custom algorithm written in the R programming language, was used to “overlap," or align and compare, the unordered gene partners identified by 490 individual fusion callers. The utilization of unordered gene partners allows for fusions to be 491 compared, even if different breakpoints were identified by individual algorithms, and to include 492 reciprocal fusions. Fusion partners identified by at least three of the seven callers are retained and 493 prioritized based on the number of contributing algorithms first and then by the number of 494 sequence reads providing evidence for each fusion. The overlap output retains annotations from 495 the individual callers, including breakpoints, distance between breakpoints, donor and acceptor 496 genes, reads of evidence, nucleotide sequence at breakpoint (if available), frequency information 497 from the database, and whether the identified fusion contains "known pathogenic fusion 498 partners". If discordant breakpoints are identified across callers for a set of fusion partners, the 
499 breakpoints with the most evidence, determined by number of supporting reads, are prioritized in 500 the output.

501 The fusions are filtered by the following steps (FIGURE 1A). Read-through events, which 502 occur between neighboring genes and are typically identified in both healthy and disease states, 503 are not expected to impact cellular functions $[12,24]$. This type of fusion prediction is a source of 504 false positive results, so we have implemented a filter that removes fusions detected between 505 genes fewer than 200,000 bases apart, that occur on the same strand and chromosome. Recurrent 506 fusions with uncertain biological significance have also been identified in normal tissues. To 507 prevent the inclusion of commonly occurring, benign fusions in our output, a PostgreSQL database 508 was used to filter commonly occurring artifactual fusions. This filter removes any expected fusion 509 artifact with greater than a 10\% frequency of detection based on our internal cohort. Lastly, to 510 ensure a high level of confidence in the identified fusions, we utilize a minimum threshold for level 511 of evidence, removing fusions that contain fewer than four reads of support from at least one 512 contributing algorithm.

513 While filtering can remove false positive results and reduces the time needed to review 514 predicted fusions, it also can remove true positive fusions in certain circumstances. To prevent the 515 inadvertent filtering of known fusions, a known fusion list was developed containing 325 pairs of 516 common fusion partners associated with cancer, as identified in COSMIC and TCGA (ADDITIONAL 517 FILE 1: TABLE S3][27, 52]. To increase sensitivity in the identification of known pathogenic fusions, 518 fusion partners that are on the known fusion list are retained as long as at least two callers have 519 identified the fusion. The ensemble pipeline also outputs a supplementary singleton fusion file, 520 containing fusions identified by a single caller that are on the known fusion list, allowing users to 521 examine low evidence fusions that may be of interest. 
To prioritize fusions that contain gene partners commonly found in the known fusion list,

523 we developed the "Gene Partner Predicted Pathogenicity Score" based on the frequency of the 524 individual partners in the known fusion list. Of the 325 fusions on the known fusion list, 38 genes 525 are present as a fusion partner $\geq 3$ times (AdDitional File 1: TABle 4, Figure S3). The most 526 common partners are $B R A F$ and $K M T 2 A$, which are present as fusion partners 28 times each. To 527 aide prediction of novel, or not well described, pathogenic fusions, we developed a score based on 528 known pathogenic gene partners. This score utilizes the frequency of partners present on the 529 known fusion list. The pathogenic frequency score ranges from 10 (most frequent) to 1 (least 530 frequent, but present at least 3 times):

$$
\text { Pathogenic Frequency Score }=10 /\left(f_{\max }-f\right)
$$

Where $f$ is the gene frequency and $f_{\max }$ is the maximum observed frequency. The following annotations are included in the ensemble results if an identified fusion contains one of the 38 common pathogenic gene partners: designation as a known pathogenic gene partner, inclusion of the frequency score (1-10), and gene type based on UniProt description [53].

A knowledge-based interpretation strategy was applied to the filtered list of fusion partners output by the pipeline, including the use of FusionHub [54], to inform clinical relevance, 537 such as diagnostic and/or prognostic information or a potential therapeutic target. Visual 538 assessment of the fusion events was performed by examining RNA-Seq BAM files with Integrated 539 Genome Viewer (IGV). Fusions were also assessed at the DNA level by IGV-based evaluation of 540 gene-specific paired end read alignments from ES or WGS BAM files, for potential evidence of 541 mapping discordance. Clinically relevant fusions were then assayed in our College of American 542 Pathologists (CAP)-accredited clinical laboratory using RT-PCR followed by Sanger sequencing of 543 the resulting products, and/or by Archer FusionPlex Solid Tumor panel (ArcherDx) for clinical 544 confirmation. 
AWS Implementation of the Ensemble Approach

(AWS) serverless environment (ADDITIONAL FILE 1: FiguRE S4). The workflow is initiated via a call

to Amazon API Gateway, which passes parameters, including the location of the input FASTQ files,

to an AWS Lambda function. The Lambda function initiates the AWS Batch job to load and

executes a custom fusion detection Docker image, which launches Arriba, CICER0, FusionMap,

552 FusionCatcher, JAFFA, MapSplice, and STAR-Fusion. We utilize the R5 family of instances for the

553 fusion detection algorithms. Due to the efficiency by which different algorithms are able to multi-

554 thread, each fusion detection tool is allocated 32 virtual CPUs (vCPUs), except for CICERO which is

555 allocated 16 vCPUs and JAFFA which is allocated 8 vCPUs. Using the described allocations, Arriba

556 completes the fastest ( $\sim 37$ minutes) for the runs completed year to date in 2020 , followed by

557 FusionMap ( $\sim 1$ hour 12 minutes), STAR-fusion ( $\sim 3$ hours 25 minutes), FusionCatcher ( $\sim 10$ hours

55835 minutes), CICERO ( $\sim 11$ hours 49 minutes), MapSplice ( $\sim 15$ hours 2 minutes), and JAFFA ( 27

559 hours 16 minutes), data is summarized in AdDITIONAL FILE 1: FiguRE S5. The results from the

560 fusion callers are sent to an AWS S3 output bucket, which invokes AWS Batch to load and execute

561 a Docker image with our overlap script upon completion. This allows for real-time examination of

562 results as each caller finishes, as the overlapping output is updated upon completion of each

563 individual caller, which is particularly advantageous given the long execution times for some of

564 the fusion callers. It is possible to examine results upon completion of the three fastest algorithms

565 within $\sim 3.5$ hours, which is of great benefit for cases necessitating fast turnaround times, and

566 complete results are made available by the next day. The overlap Docker image queries and

567 writes to an Aurora PostgreSQL database and performs all necessary filtering. The final results,

568 including annotated filtered and unfiltered fusion lists, are stored in an AWS S3 output bucket for 
569 subsequent interpretation. Code for the overlap algorithm is available at our GitHub repository

570 (https://github.com/nch-igm/nch-igm-ensemble-fusion-detection),

DOI:

571 10.5281/zenodo.3950385, and Docker images used to build the pipeline are available upon

572 request.

573

574 Data Analysis and Statistics

$575 \quad$ Figures were plotted using R version 4.0.2. Statistical analysis was performed by GraphPad

576 Prism 7.0e software. Graphical representation of fusion breakpoints and products were generated

577 using a modified version of INTEGRATE-Vis [55].

578

LIST OF ABBREVIATIONS

AWS: Amazon Web Services

CNS: Central Nervous System

ES: Exome Sequencing

FDR: False Discovery Rate

FFPE: Formalin Fixed, Paraffin Embedded

FFPM: Fusion Fragments Per Million

GSNAP: Genomic Short-read Nucleotide Alignment Program

Heme: Hematologic Diseases

HPF: High Power Field

HPC: High Performance Computing

IGM: Institute for Genomic Medicine

IGV: Integrated Genome Viewer

ITD: Internal Tandem Duplication 
NCH: Nationwide Children's Hospital

QC: Quality Control

RNA-Seq: RNA-Sequencing

sSGSEA: Single Sample Gene Set Enrichment Analysis

vCPU: virtual central processing unit

WGS: Whole Genome sequencing

\section{Declarations:}

601 Ethics approval and consent to participate:

602 This study was reviewed and approved by the Institutional Review Board (IRB) of The Research 603 Institute at Nationwide Children's Hospital. Informed consent was obtained from the patients 604 and/or parents for molecular genetic analysis, which included RNA-sequencing. These protocols 605 allowed for return of results from research sequencing studies after confirmation in a CLIAcertified laboratory.

Availability of data and materials

609 DNA and RNA sequencing data for this study has been deposited to dbGAP, accession number $610 \quad$ phs001820.v1.p1

611 Seraseq fastq files, from the benchmarking studies, have been deposited to the NIH Sequence Read 612 Archive (SRA), accession number PRJNA679580.

613 Code for the overlap algorithm is available at our GitHub repository (https://github.com/nch614 igm/nch-igm-ensemble-fusion-detection), DOI: 10.5281/zenodo.3950385

615 The Docker image used to run the overlap algorithm is available upon request for running the 616 ensemble pipeline in an AWS serverless environment. 
617 The Docker image used to run previously published fusion detection algorithms is also available 618 upon request for running the ensemble pipeline in an AWS serverless environment.

621 No Competing interests: Stephanie LaHaye, James Fitch, Kyle Voytovich, Adam Herman, 622 Benjamin Kelly, Grant Lammi, Saranga Wijeratne, Kathleen Schieffer, Natalie Bir, Sean McGrath, 623 Anthony Miller, Amy Wetzel, Katherine Miller, Tracy Bedrosian, Kristen Leraas, Ajay Gupta, 624 Bhuvana Setty, Jeffrey Leonard, Jonathan Finlay, Mohamed Abdelbaki, Diana Osorio, Selene Koo, 625 Daniel Koboldt, Vincent Magrini, Catherine Cottrell, Richard Wilson and Peter White.

626 Elaine Mardis: Qiagen N.V., supervisory board member, honorarium and stock-based 627 compensation.

628 Daniel Boué: Illumina (ILMN) share holder.

Funding:

631 We thank the Nationwide Children's Foundation and The Abigail Wexner Research Institute at 632 Nationwide Children's Hospital for generously supporting this body of work. These funding bodies 633 had no role in the design of the study, no role in the collection, analysis, and interpretation of data 634 and no role in writing the manuscript.

Authors' contributions:

637 SL analyzed and interpreted fusion data, contributed to development of overlap algorithm and 638 Docker images, contributed to AWS serverless workflow, and wrote the manuscript. JF and KV 639 contributed development of overlap algorithm and Docker images, contributed to AWS serverless 640 workflow, and contributed to manuscript writing. AH, BJK, GL, and SW provided data analysis 
641 support, designed AWS serverless workflow, and contributed to manuscript writing. SF

642 contributed to manuscript revisions and oversaw, organized, and performed data upload to SRA.

643 KMS contributed to analysis and interpretation of RNA-Seq results and performed clinical data

644 acquisition. KM, TAB, KL and DK provided NGS analysis and interpretation for cancer cohort data.

645 NB, SDM, and ARM perform library preparations and developed laboratory procedures for RNA-

646 Seq processing/QC. AW managed RNA-Seq processing and analysis. KL managed and coordinated

647 all clinical samples. DRB, JRL, JLF, MA, DSO, AG, BS, and SCK contributed to the enrollment of

648 patients onto the NCH cancer protocols and provided clinical expertise, DRB and SCK also

649 contributed pathology materials (fixed or frozen tissues etc.) following QA and/or QC reviews of

650 enrollee pathology. VM oversaw technology development and contributed to the conceptual

651 design of project. CEC, ERM, and RKW developed, led, and supervised work performed on cancer

652 protocol, contributed to conceptual design of project, contributed to analysis and interpretation of

653 RNA-Seq results, and contributed to manuscript writing and revision. PW conceived, designed,

654 and supervised the project, oversaw and contributed to algorithm development, provided support

655 for utilization of AWS and computational resources, and contributed significantly to manuscript

656 writing and revision. All authors read and approved the final manuscript.

658 Acknowledgements

659 We thank the patients and their families for participating in our translational research protocol.

660 We thank the Nationwide Foundation Pediatric Innovation Fund for generously supporting this 661 project.

662

663

664 
REFERENCES

666

667

668

669

670

671

672

673

674

675

676

677

678

679

680

681

682

683

684

685

686

687

688

689

690

691

692

693

694

695

696

697

698

699

700

701

702

703

704

705

706

707

708

709

710

1. Steliarova-Foucher E, Colombet M, Ries LAG, Moreno F, Dolya A, Bray F, Hesseling P, Shin HY, Stiller CA, contributors I-: International incidence of childhood cancer, 2001-10: a population-based registry study. Lancet Oncol 2017, 18:719-731.

2. Amatu A, Sartore-Bianchi A, Siena S: NTRK gene fusions as novel targets of cancer therapy across multiple tumour types. ESMO Open 2016, 1:e000023.

3. Pui CH, Gajjar AJ, Kane JR, Qaddoumi IA, Pappo AS: Challenging issues in pediatric oncology. Nat Rev Clin Oncol 2011, 8:540-549.

4. $\quad$ Siegel RL, Miller KD, Jemal A: Cancer statistics, 2016. CA Cancer J Clin 2016, 66:7-30.

5. Vogelstein B, Papadopoulos N, Velculescu VE, Zhou S, Diaz LA, Jr., Kinzler KW: Cancer genome landscapes. Science 2013, 339:1546-1558.

6. Grobner SN, Worst BC, Weischenfeldt J, Buchhalter I, Kleinheinz K, Rudneva VA, Johann PD, Balasubramanian GP, Segura-Wang M, Brabetz S, et al: The landscape of genomic alterations across childhood cancers. Nature 2018, 555:321-327.

7. Marshall GM, Carter DR, Cheung BB, Liu T, Mateos MK, Meyerowitz JG, Weiss WA: The prenatal origins of cancer. Nat Rev Cancer 2014, 14:277-289.

8. Rowley JD: Letter: A new consistent chromosomal abnormality in chronic myelogenous leukaemia identified by quinacrine fluorescence and Giemsa staining. Nature 1973, 243:290-293.

9. Soda M, Choi YL, Enomoto M, Takada S, Yamashita Y, Ishikawa S, Fujiwara S, Watanabe H, Kurashina K, Hatanaka H, et al: Identification of the transforming EML4-ALK fusion gene in non-small-cell lung cancer. Nature 2007, 448:561-566.

10. Jia Y, Xie Z, Li H: Intergenically Spliced Chimeric RNAs in Cancer. Trends Cancer 2016, 2:475-484.

11. Li Y, Li Y, Yang T, Wei S, Wang J, Wang M, Wang Y, Zhou Q, Liu H, Chen J: Clinical significance of EML4-ALK fusion gene and association with EGFR and KRAS gene mutations in 208 Chinese patients with non-small cell lung cancer. PLOS One 2013, 8:e52093.

12. Dupain C, Harttrampf AC, Urbinati G, Geoerger B, Massaad-Massade L: Relevance of Fusion Genes in Pediatric Cancers: Toward Precision Medicine. Mol Ther Nucleic Acids 2017, 6:315-326.

13. Bernt KM, Hunger SP: Current concepts in pediatric Philadelphia chromosomepositive acute lymphoblastic leukemia. Front Oncol 2014, 4:54.

14. Hawkins C, Walker E, Mohamed N, Zhang C, Jacob K, Shirinian M, Alon N, Kahn D, Fried I, Scheinemann K, et al: BRAF-KIAA1549 fusion predicts better clinical outcome in pediatric low-grade astrocytoma. Clin Cancer Res 2011, 17:4790-4798.

15. Park SH, Won J, Kim SI, Lee Y, Park CK, Kim SK, Choi SH: Molecular Testing of Brain Tumor. J Pathol Transl Med 2017, 51:205-223.

16. Yuan L, Liu ZH, Lin ZR, Xu LH, Zhong Q, Zeng MS: Recurrent FGFR3-TACC3 fusion gene in nasopharyngeal carcinoma. Cancer Biol Ther 2014, 15:1613-1621.

17. Jones DT, Kocialkowski S, Liu L, Pearson DM, Backlund LM, Ichimura K, Collins VP: Tandem duplication producing a novel oncogenic BRAF fusion gene defines the majority of pilocytic astrocytomas. Cancer Res 2008, 68:8673-8677.

18. Morris SW, Kirstein MN, Valentine MB, Dittmer K, Shapiro DN, Look AT, Saltman DL: Fusion of a kinase gene, ALK, to a nucleolar protein gene, NPM, in non-Hodgkin's lymphoma. Science 1995, 267:316-317. 
19. Mosse YP, Lim MS, Voss SD, Wilner K, Ruffner K, Laliberte J, Rolland D, Balis FM, Maris JM, Weigel BJ, et al: Safety and activity of crizotinib for paediatric patients with refractory solid tumours or anaplastic large-cell lymphoma: a Children's Oncology Group phase 1 consortium study. Lancet Oncol 2013, 14:472-480.

20. Huddleston J, Chaisson MJP, Steinberg KM, Warren W, Hoekzema K, Gordon D, GravesLindsay TA, Munson KM, Kronenberg ZN, Vives L, et al: Discovery and genotyping of structural variation from long-read haploid genome sequence data. Genome Res 2017, 27:677-685.

21. Nattestad M, Goodwin S, Ng K, Baslan T, Sedlazeck FJ, Rescheneder P, Garvin T, Fang H, Gurtowski J, Hutton E, et al: Complex rearrangements and oncogene amplifications revealed by long-read DNA and RNA sequencing of a breast cancer cell line. Genome Res 2018, 28:1126-1135.

22. Maher CA, Kumar-Sinha C, Cao X, Kalyana-Sundaram S, Han B, Jing X, Sam L, Barrette T, Palanisamy N, Chinnaiyan AM: Transcriptome sequencing to detect gene fusions in cancer. Nature 2009, 458:97-101.

23. Wang $Q$, Xia J, Jia $P$, Pao W, Zhao Z: Application of next generation sequencing to human gene fusion detection: computational tools, features and perspectives. Brief Bioinform 2013, 14:506-519.

24. He Y, Yuan C, Chen L, Lei M, Zellmer L, Huang H, Liao DJ: Transcriptional-Readthrough RNAs Reflect the Phenomenon of "A Gene Contains Gene(s)" or "Gene(s) within a Gene" in the Human Genome, and Thus Are Not Chimeric RNAs. Genes (Basel) 2018, 9.

25. Haas BJ, Dobin A, Li B, Stransky N, Pochet N, Regev A: Accuracy assessment of fusion transcript detection via read-mapping and de novo fusion transcript assembly-based methods. Genome Biol 2019, 20:213.

26. Liu S, Tsai WH, Ding Y, Chen R, Fang Z, Huo Z, Kim S, Ma T, Chang TY, Priedigkeit NM, et al: Comprehensive evaluation of fusion transcript detection algorithms and a metacaller to combine top performing methods in paired-end RNA-seq data. Nucleic Acids Res 2016, 44:e47.

27. Gao Q, Liang WW, Foltz SM, Mutharasu G, Jayasinghe RG, Cao S, Liao WW, Reynolds SM, Wyczalkowski MA, Yao L, et al: Driver Fusions and Their Implications in the Development and Treatment of Human Cancers. Cell Rep 2018, 23:227-238 e223.

28. Church AJ, Calicchio ML, Nardi V, Skalova A, Pinto A, Dillon DA, Gomez-Fernandez CR, Manoj N, Haimes JD, Stahl JA, et al: Recurrent EML4-NTRK3 fusions in infantile fibrosarcoma and congenital mesoblastic nephroma suggest a revised testing strategy. Mod Pathol 2018, 31:463-473.

29. Consortium ITP-CAoWG: Pan-cancer analysis of whole genomes. Nature 2020, 578:8293.

30. Stransky N, Cerami E, Schalm S, Kim JL, Lengauer C: The landscape of kinase fusions in cancer. Nat Commun 2014, 5:4846.

31. International Cancer Genome Consortium PedBrain Tumor P: Recurrent MET fusion genes represent a drug target in pediatric glioblastoma. Nat Med 2016, 22:1314-1320.

32. Torre M, Jessop N, Hornick JL, Alexandrescu S: Expanding the spectrum of pediatric NTRK-rearranged fibroblastic tumors to the central nervous system: A case report with RBPMS-NTRK3 fusion. Neuropathology 2018, 38:624-630.

33. Flucke U, van Noesel MM, Wijnen M, Zhang L, Chen CL, Sung YS, Antonescu CR: TFG-MET fusion in an infantile spindle cell sarcoma with neural features. Genes Chromosomes Cancer 2017, 56:663-667. 
34. Cocco E, Scaltriti M, Drilon A: NTRK fusion-positive cancers and TRK inhibitor therapy. Nat Rev Clin Oncol 2018, 15:731-747.

35. Pekmezci M, Villanueva-Meyer JE, Goode B, Van Ziffle J, Onodera C, Grenert JP, Bastian BC, Chamyan G, Maher OM, Khatib Z, et al: The genetic landscape of ganglioglioma. Acta Neuropathol Commun 2018, 6:47.

36. Yoshihara K, Wang Q, Torres-Garcia W, Zheng S, Vegesna R, Kim H, Verhaak RG: The landscape and therapeutic relevance of cancer-associated transcript fusions. Oncogene 2015, 34:4845-4854.

37. Bar EE, Lin A, Tihan T, Burger PC, Eberhart CG: Frequent gains at chromosome 7q34 involving BRAF in pilocytic astrocytoma. J Neuropathol Exp Neurol 2008, 67:878-887.

38. Lin A, Rodriguez FJ, Karajannis MA, Williams SC, Legault G, Zagzag D, Burger PC, Allen JC, Eberhart CG, Bar EE: BRAF alterations in primary glial and glioneuronal neoplasms of the central nervous system with identification of 2 novel KIAA1549:BRAF fusion variants. J Neuropathol Exp Neurol 2012, 71:66-72.

39. Yamashita S, Takeshima H, Matsumoto F, Yamasaki K, Fukushima T, Sakoda H, Nakazato M, Saito K, Mizuguchi A, Watanabe T, et al: Detection of the KIAA1549-BRAF fusion gene in cells forming microvascular proliferations in pilocytic astrocytoma. PLoS One 2019, 14:e0220146.

40. Jones DT, Hutter B, Jager N, Korshunov A, Kool M, Warnatz HJ, Zichner T, Lambert SR, Ryzhova M, Quang DA, et al: Recurrent somatic alterations of FGFR1 and NTRK2 in pilocytic astrocytoma. Nat Genet 2013, 45:927-932.

41. Tian L, Li Y, Edmonson MN, Zhou X, Newman S, McLeod C, Thrasher A, Liu Y, Tang B, Rusch $\mathrm{MC}$, et al: CICERO: a versatile method for detecting complex and diverse driver fusions using cancer RNA sequencing data. Genome Biol 2020, 21:126.

42. Berger AC, Korkut A, Kanchi RS, Hegde AM, Lenoir W, Liu W, Liu Y, Fan H, Shen H, Ravikumar V, et al: A Comprehensive Pan-Cancer Molecular Study of Gynecologic and Breast Cancers. Cancer Cell 2018, 33:690-705 e699.

43. Dobin A, Davis CA, Schlesinger F, Drenkow J, Zaleski C, Jha S, Batut P, Chaisson M, Gingeras TR: STAR: ultrafast universal RNA-seq aligner. Bioinformatics 2013, 29:15-21.

44. Anders S, Pyl PT, Huber W: HTSeq--a Python framework to work with high-throughput sequencing data. Bioinformatics 2015, 31:166-169.

45. Love MI, Huber W, Anders S: Moderated estimation of fold change and dispersion for RNA-seq data with DESeq2. Genome Biol 2014, 15:550.

46. Subramanian A, Tamayo P, Mootha VK, Mukherjee S, Ebert BL, Gillette MA, Paulovich A, Pomeroy SL, Golub TR, Lander ES, Mesirov JP: Gene set enrichment analysis: a knowledge-based approach for interpreting genome-wide expression profiles. ProC Natl Acad Sci U S A 2005, 102:15545-15550.

47. Seraseq Tumor Fusion RNA Mix3 [https://www.seracare.com/globalassets/seracareresources/pr-0710-0431-seraseq-tumor-fusion-rna-mix-v3-10330722.pdf]

48. Ge H, Liu K, Juan T, Fang F, Newman M, Hoeck W: FusionMap: detecting fusion genes from next-generation sequencing data at base-pair resolution. Bioinformatics 2011, 27:1922-1928.

49. Davidson NM, Majewski IJ, Oshlack A: JAFFA: High sensitivity transcriptome-focused fusion gene detection. Genome Med 2015, 7:43.

50. Nicorici D, S atalan M, Edgren H, Kangaspeska s, Murum“agi A, Kallioniemi o, Virtanen S, Kilkku 0: FusionCatcher - a tool for finding somatic fusion genes in paired-end RNAsequencing data. bioRxiv 2014. 
805 51. Wang K, Singh D, Zeng Z, Coleman SJ, Huang Y, Savich GL, He X, Mieczkowski P, Grimm SA, Perou CM, et al: MapSplice: accurate mapping of RNA-seq reads for splice junction discovery. Nucleic Acids Res 2010, 38:e178.

52. Tate JG, Bamford S, Jubb HC, Sondka Z, Beare DM, Bindal N, Boutselakis H, Cole CG, Creatore C, Dawson E, et al: COSMIC: the Catalogue Of Somatic Mutations In Cancer. Nucleic Acids Res 2019, 47:D941-D947.

53. UniProt C: UniProt: the universal protein knowledgebase in 2021. Nucleic Acids Res 2020.

54. Panigrahi P, Jere A, Anamika K: FusionHub: A unified web platform for annotation and visualization of gene fusion events in human cancer. PLOS One 2018, 13:e 0196588. 
FigURE 1
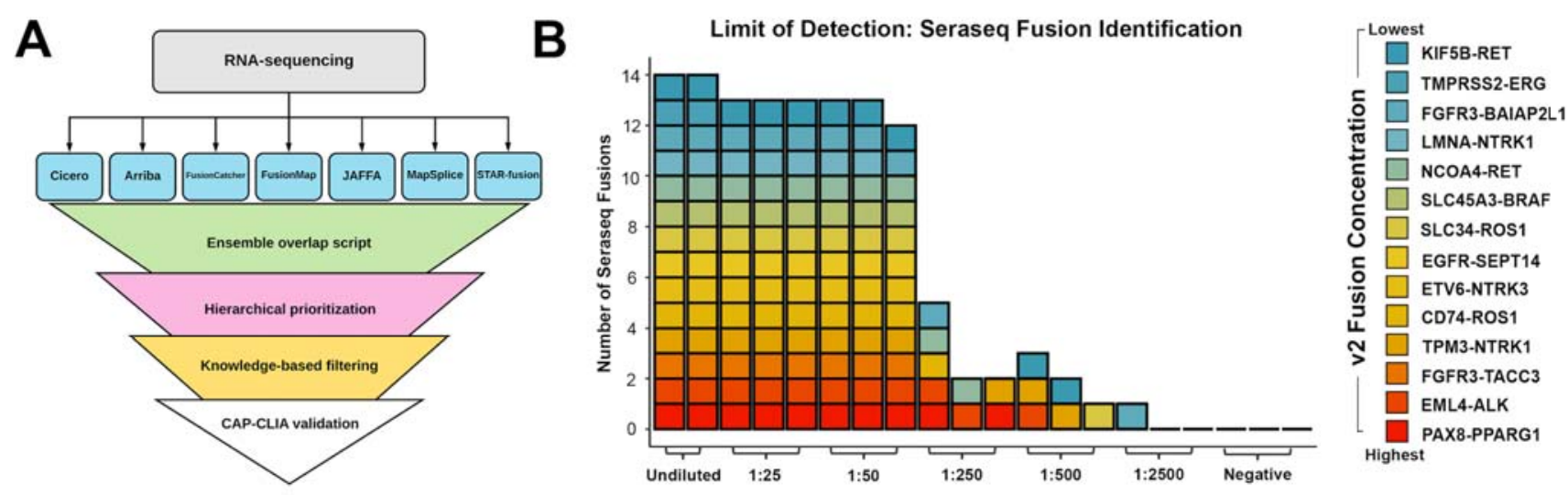

Figure 1. The ensemble fusion detection pipeline identifies true positive fusions. A) The

ensemble approach identifies fusions in RNA-Seq data by overlapping results from Arriba,

CICERO, FusionCatcher, FusionMap, JAFFA, MapSplice, and STAR-Fusion. It hierarchically

prioritizes and filters the fusions utilizing an in-house PostgreSQL database and knowledge base,

prior to producing an output list of predicted fusions. In many cases, detected fusions were

orthogonally tested by clinical confirmation in order to return a medically meaningful result. B)

The ensemble pipeline was tested on a dilution series of a reference control reagent (SeraCare) to

determine sensitivity and limit of detection. We optimized the pipeline using the undiluted

reference control reagent, identifying that by requiring $\geq 3$ callers to have overlap for a detected

fusion, and by utilizing filtering of known false positive fusion calls and cross-referencing a list of

831 known fusions, all 14 fusions were identified. Colors representing different fusions present in the

832 SeraSeq v2 reagent are ordered by their absolute proportions. We then applied the optimized

833 pipeline to the dilution series, showing that the numbers of identified fusions were reduced in 

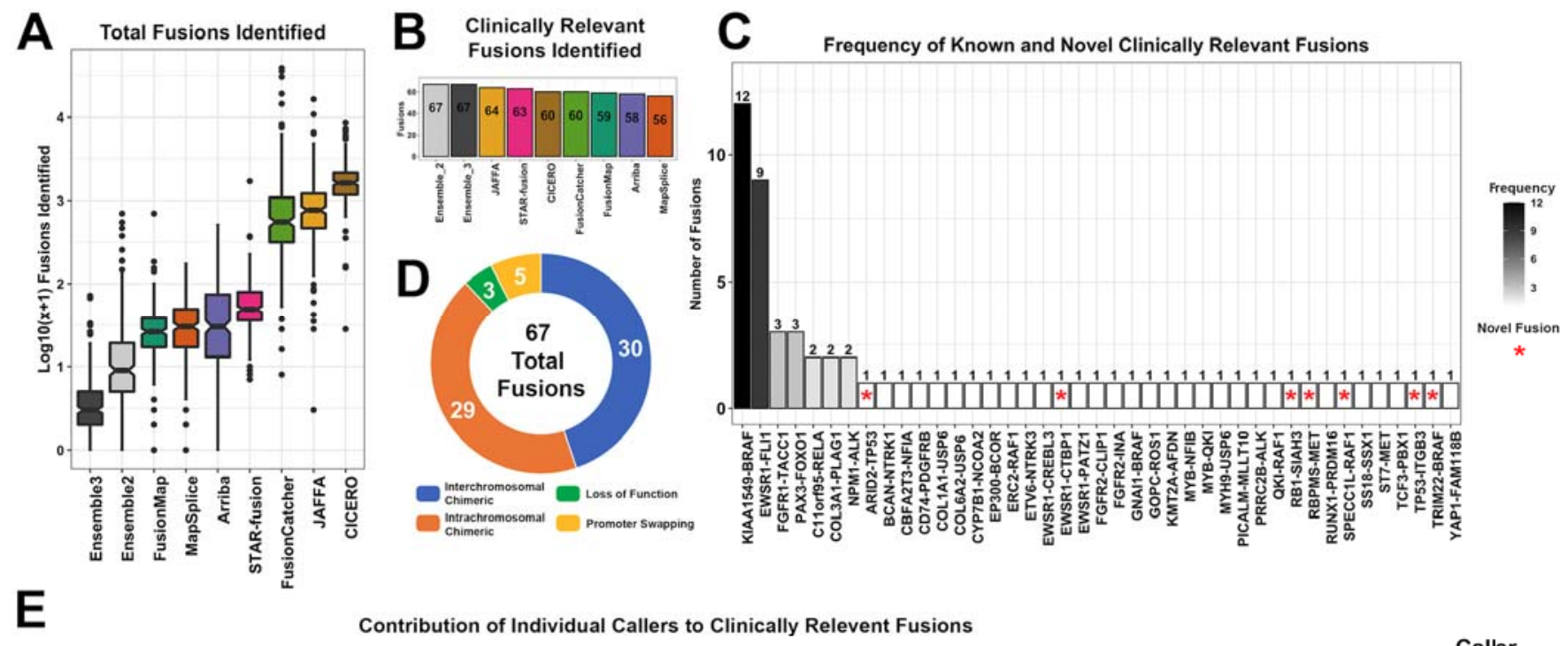

Figure 2. Clinically relevant fusions identified by the ensemble approach in a pediatric

cancer and hematologic disease cohort. A) The ensemble approach, with automated filtering,

identifies significantly fewer fusions compared to individual callers. The number of fusions is

841 plotted as $\log _{10}(x+1)$ to account for 0 fusions identified in some cases. Callers are sorted by the

842 lowest median number of fusions identified to highest.. B) 67 Clinically relevant fusions were

843 identified, represented as a bar graph with decreasing fusions per individual algorithm,

844 highlighting the sensitivity of the ensemble approach compared to individual algorithms. No

845 individual algorithm was able to identify all 67 fusions. C) Of the 67 clinically relevant fusions

846 identified, 30 were interchromosomal chimeric (blue), 29 were intrachromosomal chimeric

847 (orange), 3 were loss of function (green), and 5 were promoter swapping (yellow) fusions. D) Of

848 the 67 clinically relevant fusions identified, 7 are novel events (red asterisk), while the remaining

84960 fusion partners had been described previously in the literature. E) A stacked bar graph

850 represents the individual fusion callers that contributed to each clinically relevant fusion. 


\section{FIGURE 3}
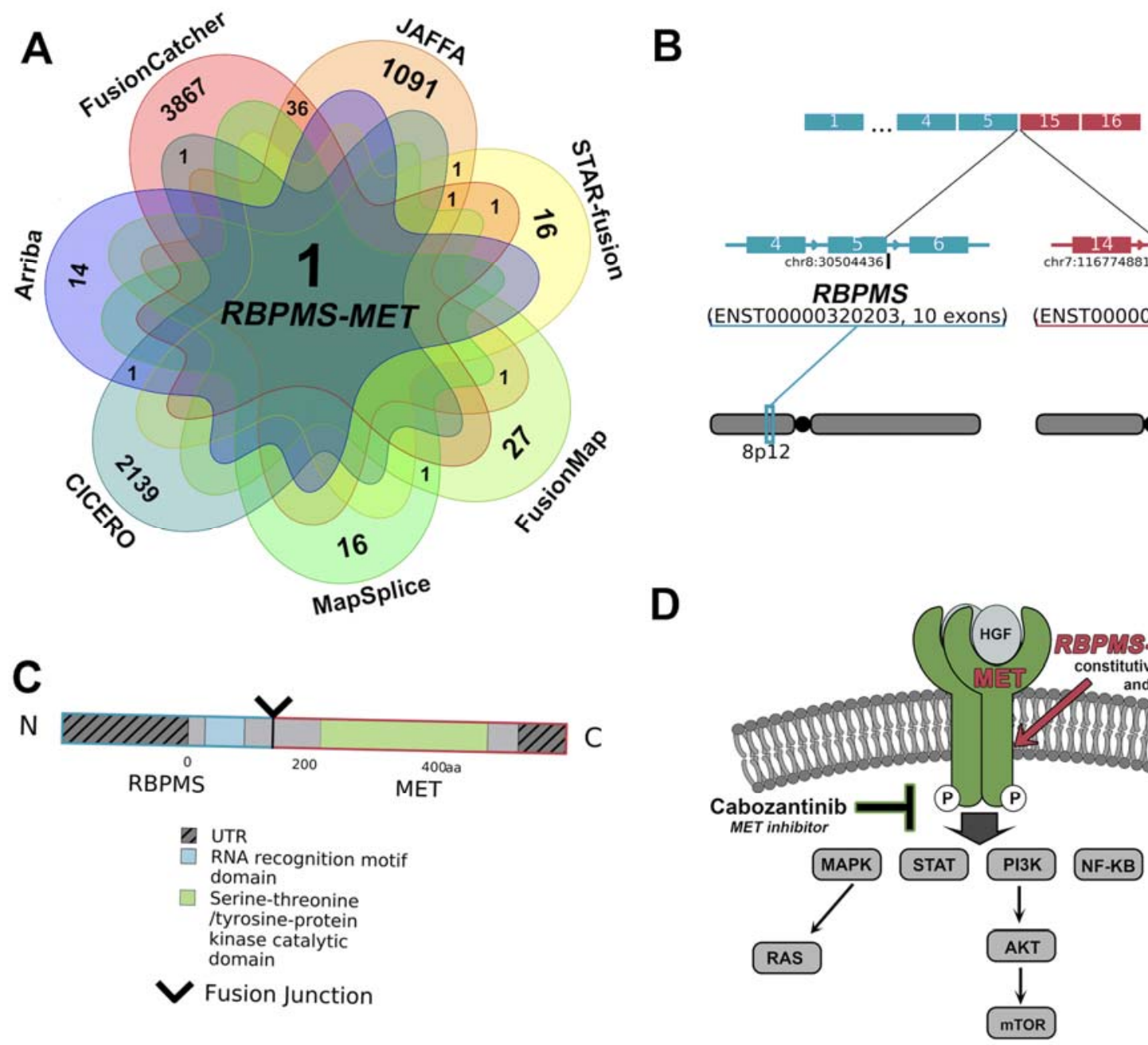

Figure 3. An RBPMS-MET fusion identified in a patient with an infantile fibrosarcoma-like

tumor. A) RBPMS-MET fusion was identified by all seven fusion callers in the filtered overlap results. The number of fusions identified by each caller is in the outer VENN diagram sections, while internal numbers indicate overlapping fusions found post-filtering ( 0 overlaps between callers are not shown). B) The RBPMS-MET fusion is an interchromosomal event, occurring between 8p12 and 7q31.2 and joining exon 5 of RBPMS (blue) to exon 15 of MET (red). C) The

859 fusion protein product includes the RNA recognition motif domain of RBPMS and the tyrosine 860 kinase catalytic domain of MET. D) The RBPMS-MET fusion is predicted to cause constitutive 861 phosphorylation and activation of MET, targetable using cabozantinib. 


\section{FigURE 4}

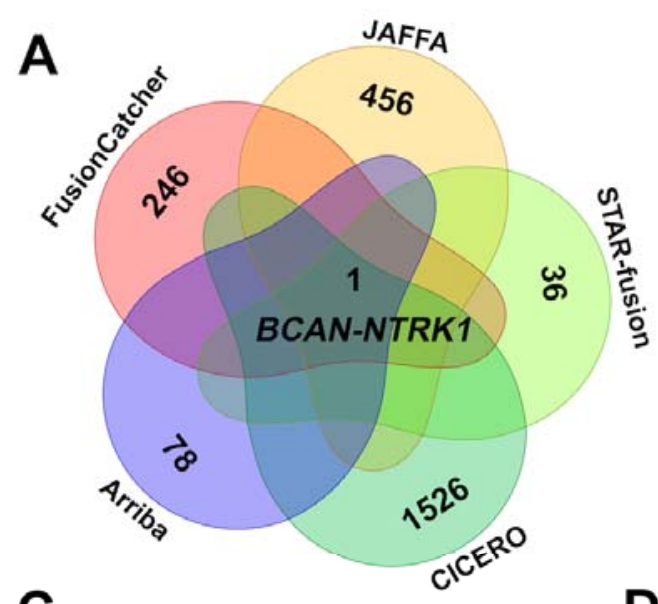

C
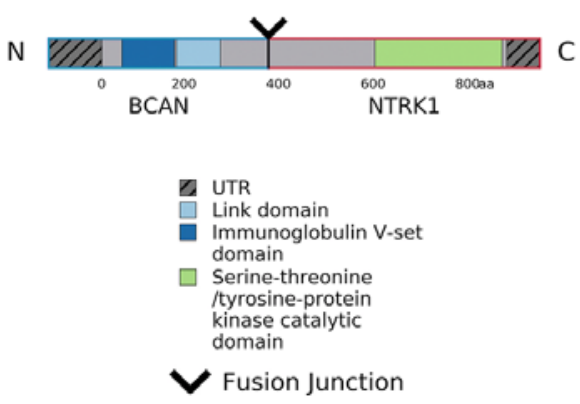

863

864

865

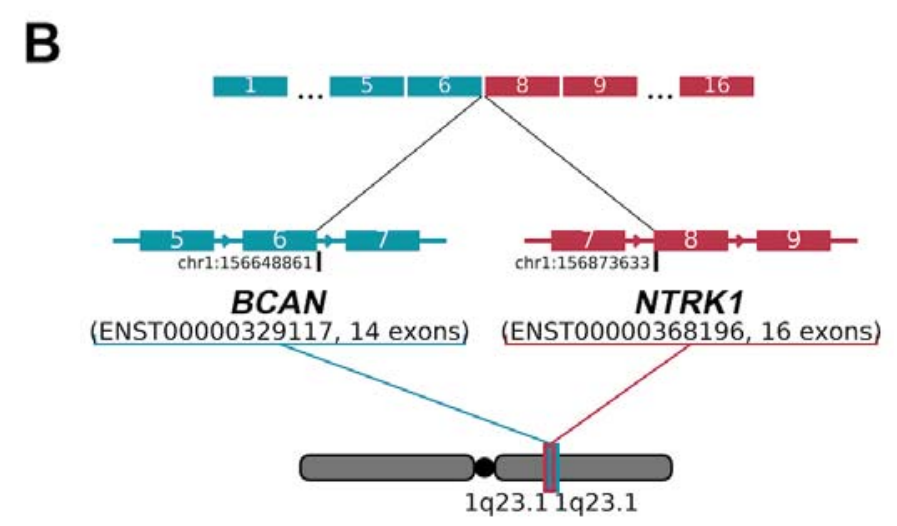

D

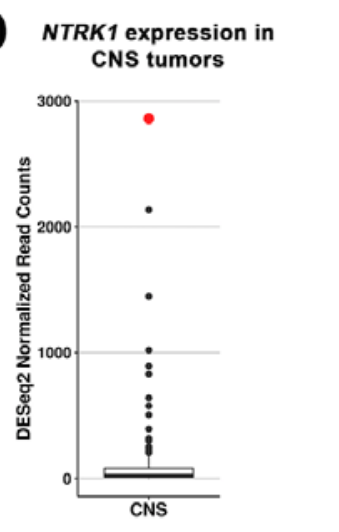

Figure 4. Targetable NTRK1 fusion identified in an infiltrating glioma. A) The BCAN-NTRK1 fusion was identified by 5 of 7 fusion callers, and was the only fusion returned by the filtered overlap results. Total fusions identified by each caller are shown, FusionMap and MapSplice identified no overlapping fusions that passed filtering ( 0 overlaps between callers are not shown). B) The BCAN-NTRK1 fusion is an intrachromosomal event occurring on 1q23.1, joining exon 6 of BCAN (blue) and exon 8 of NTRK1 (red). C) This fusion results in the juxtaposition of the tyrosine kinase catalytic domain of the NTRK1 gene to the 5' end of the BCAN gene. D) NTRK1 is highly expressed in this patient (red) compared to CNS tumors (black) in the NCH cohort (CNS tumors: $\mathrm{n}$ $=138$ ), with a normalized read count that is 7.70 standard deviations above the mean (131.2). E) The BCAN-NTRK1 fusion is predicted to increase expression and activation of the tyrosine kinase NTRK1, which may be inhibited by TRK inhibitor therapy (green). 


\section{FIGURE 5}
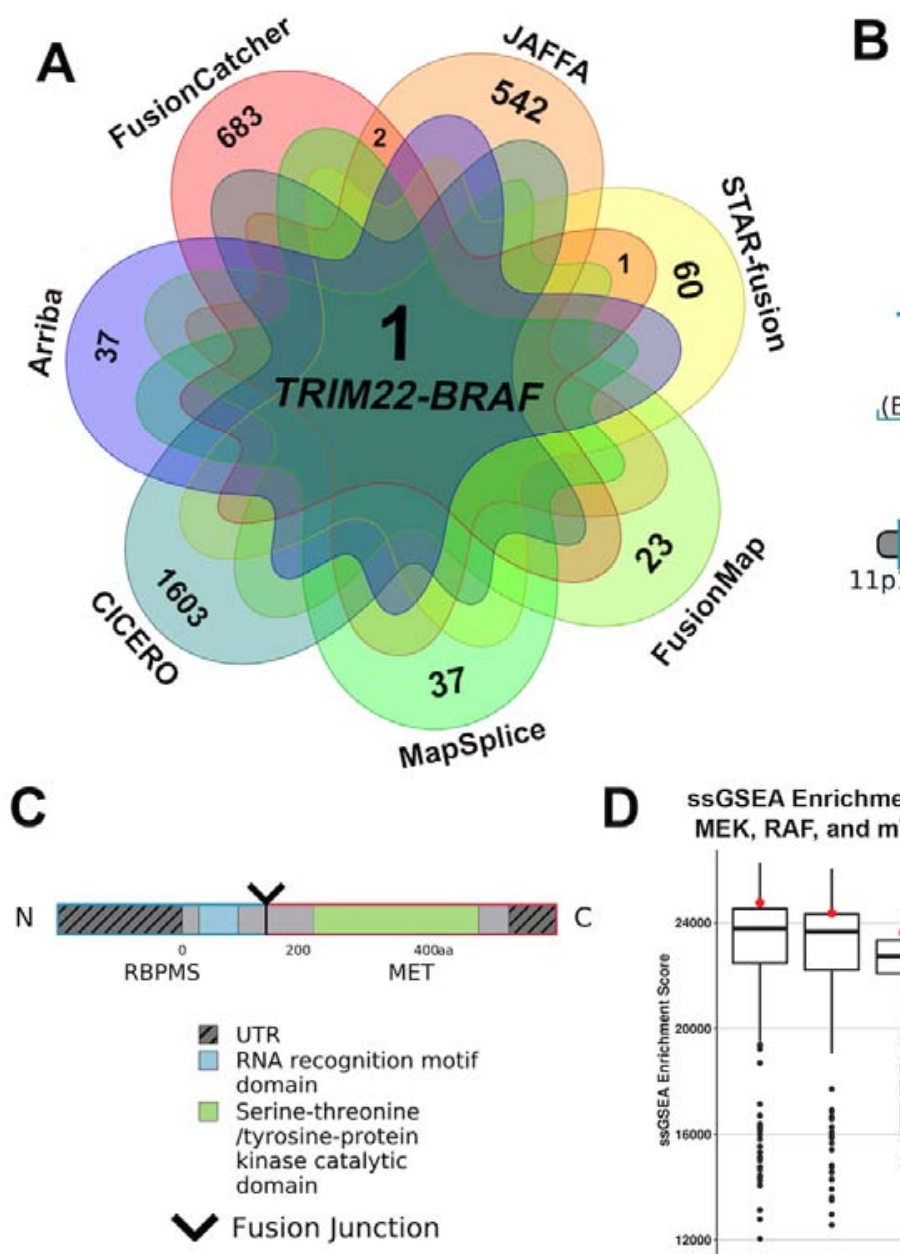

D
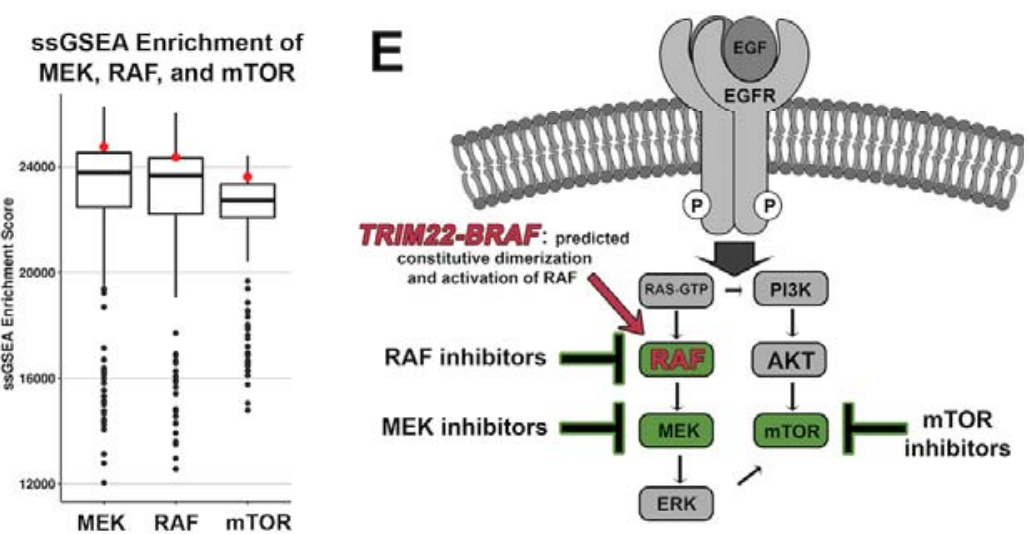

Figure 5. Identification of a novel $B R A F$ fusion in a mixed neuronal-glial tumor. A) The TRIM22-BRAF fusion was identified by all seven fusion callers and in the filtered overlap results, total fusions identified by each caller and overlapping fusions are shown (0 overlaps between callers are not shown). B) The TRIM22-BRAF fusion is an interchromosomal event between 11p15.4 and 7q34, joining exon 2 of TRIM22 (blue) to exon 9 of BRAF (red). C) The resulting fusion product contains the 5' TRIM22 zinc finger binding domains and BRAF tyrosine kinase catalytic domain. D) Single sample gene set enrichment analysis (sSGSEA) indicates a trend toward an enrichment of the MEK (above the $75^{\text {th }}$ percentile, 0.68 standard deviations above the mean of 22756.87), RAF (above the $75^{\text {th }}$ percentile, 0.60 standard deviations above the mean of 22635.74), and mTOR (above the $75^{\text {th }}$ percentile, 0.72 standard deviations above the mean of 22191.50) upregulated gene sets in the TRIM22-BRAF sample (red) compared to the pan-cancer NCH cohort (black) (pan-cancer cohort: $\mathrm{n}=229$ ). E) The TRIM22-BRAF fusion is predicted to cause constitutive dimerization and activation of the BRAF kinase domain, shown in D), which could be targeted by RAF, MEK, and mTOR inhibitors (green). 


\section{TABLE 1}

892

\begin{tabular}{|c|c|c|c|c|c|}
\hline Tool & Version & Aligner & Reference & $\begin{array}{c}\text { Average } \\
\text { Fusions Called } \\
\text { per Case }\end{array}$ & $\begin{array}{c}\text { Sensitivity } \\
\text { (Clinically Relevant } \\
\text { Fusions Called } \\
\text { out of 67) } \\
\end{array}$ \\
\hline Arriba & v1.2.0 & STAR aligner & $\begin{array}{c}\text { Haas et al, } \\
2019 \text { Genome } \\
\text { Biol }\end{array}$ & 54 & $86.6 \%(58)$ \\
\hline CICERO & v0.3.0 & $\begin{array}{l}\text { candidate SV (structural } \\
\text { variant) breakpoints and } \\
\text { splice junction }\end{array}$ & $\begin{array}{c}\text { Tian et al., } \\
2020 \text { Genome } \\
\text { Biol }\end{array}$ & 1915 & $89.6 \%(60)$ \\
\hline FusionMap & $\begin{array}{l}\text { v mono- } \\
2.10 .9\end{array}$ & $\begin{array}{l}\text { GSNAP (Genomic Short- } \\
\text { read Nucleotide } \\
\text { Alignment Program) - } \\
12 \text { mer based }\end{array}$ & $\begin{array}{c}\text { Ge et al., } 2011 \\
\text { Bioinformatics }\end{array}$ & 34 & $88.1 \%(59)$ \\
\hline FusionCatcher & v0.99.7c & $\begin{array}{c}4 \text { aligners to identify } \\
\text { junctions (Bowtie, BLAT, } \\
\text { STAR, and Bowtie2) }\end{array}$ & $\begin{array}{l}\text { Nicorici et al., } \\
2014 \text { bioRxiv }\end{array}$ & 1558 & $89.6 \%(60)$ \\
\hline JAFFA & $\begin{array}{l}\text { direct } \\
\text { v1.09 }\end{array}$ & $\begin{array}{l}\text { BLAT, uses kmers to } \\
\text { selects reads that do not } \\
\text { map to known transcripts }\end{array}$ & $\begin{array}{l}\text { Davidson et al, } \\
2015 \text { Genome } \\
\text { Med }\end{array}$ & 1141 & $95.5 \%(64)$ \\
\hline MapSplice & v2.2.1 & $\begin{array}{l}\text { approximate sequence } \\
\text { alignment combined with } \\
\text { a local search }\end{array}$ & $\begin{array}{l}\text { Wang et al, } \\
2010 \text { Nucleic } \\
\text { Acids Res }\end{array}$ & 37 & $83.6 \%(56)$ \\
\hline STAR-fusion & v1.6.0 & STAR aligner & $\begin{array}{l}\text { Haas et al, } \\
2019 \text { Genome } \\
\text { Biol }\end{array}$ & 72 & $94.0 \%(63)$ \\
\hline
\end{tabular}

Table 1. Performance comparison of individual fusion calling algorithms. Fusion calling 


\begin{tabular}{ccccc}
\hline Algorithm & $\begin{array}{c}\text { Total } \\
\text { Fusions } \\
\text { Identified }\end{array}$ & $\begin{array}{c}\text { Seraseq } \\
\text { Fusions } \\
\text { Identified }\end{array}$ & Sensitivity & Precision \\
\hline Arriba & 23.5 & 13 & $92.9 \%$ & $55.3 \%$ \\
MapSplice & 22 & 12 & $85.7 \%$ & $54.6 \%$ \\
STAR-fusion & 32 & 14 & $100.0 \%$ & $43.6 \%$ \\
FusionMap & 30 & 12.5 & $89.3 \%$ & $41.7 \%$ \\
FusionCatcher & 299.5 & 13 & $92.9 \%$ & $4.3 \%$ \\
JAFFA & 470.5 & 12.5 & $89.3 \%$ & $2.7 \%$ \\
CICER0 & 1323 & 14 & $100.0 \%$ & $1.1 \%$ \\
\hline $\begin{array}{c}\text { Ensemble 2 callers } \\
\text { Ensemble 2 callers } \\
+ \text { filter }\end{array}$ & 38.5 & 14 & $100.0 \%$ & $36.4 \%$ \\
$\begin{array}{c}\text { Ensemble 2 callers } \\
+ \text { filter + known fusion list }\end{array}$ & 15.5 & 12 & $85.7 \%$ & $77.4 \%$ \\
\hline $\begin{array}{c}\text { Ensemble 3 callers } \\
\text { Ensemble 3 callers } \\
+ \text { f filter }\end{array}$ & 17.5 & 14 & $100.0 \%$ & $80.0 \%$ \\
\hline $\begin{array}{c}\text { Ensemble 3 callers } \\
+ \text { filter + known fusion list }\end{array}$ & 15 & 14 & $100.0 \%$ & $93.3 \%$ \\
\hline$\quad 12$ & 12 & $85.7 \%$ & $100.0 \%$ \\
\hline & 14 & $100.0 \%$ & $100.0 \%$ \\
\hline
\end{tabular}

900 Table 2. Improved precision in fusion detection, utilizing Seraseq controls, achieved

901 through utilization of the ensemble pipeline. Data shown is from undiluted Seraseq v3 RNA-

902 Seq, experiments performed in duplicate, averages are shown. Individual algorithms are listed by

903 precision, in descending order. Seraseq fusions identified (true positive) are out of a possible 14

904 fusions. 